\title{
Barium even-to-odd isotope abundance ratios in thick disk and thin disk stars ${ }^{\star}$
}

\author{
L. Mashonkina ${ }^{1,2,3}$ and G. Zhao ${ }^{3}$ \\ 1 Institut für Astronomie und Astrophysik der Universität München, Scheinerstr. 1, 81679 München, Germany \\ e-mail: 1yuda@usm.1mu.de \\ 2 Institute of Astronomy, Russian Academy of Science, Pyatnitskaya 48, 119017 Moscow, Russia \\ e-mail: lima@inasan.ru \\ 3 National Astronomical Observatories, Chinese Academy of Science, A20 Datun Road, Chaoyang District, Beijing 100012 , \\ PR China
}

Received 22 December 2005 / Accepted 26 March 2006

\section{ABSTRACT}

\begin{abstract}
We present the $\mathrm{Ba}$ even-to-odd isotope abundance ratios in $25 \mathrm{cool}$ dwarf stars with the metallicity $[\mathrm{Fe} / \mathrm{H}]$ ranging between 0.25 and -1.35 . Our method takes advantage of the hyperfine structure (HFS) affecting the Ba II resonance line of the odd isotopes. The fractional abundance of the odd isotopes of $\mathrm{Ba}$ is derived from a requirement that $\mathrm{Ba}$ abundances from the resonance line $\lambda 4554$ and subordinate lines $\lambda 5853$ and $\lambda 6496$ must be equal. The results are based on NLTE line formation and analysis of high resolution ( $R \sim 60000)$ high signal-to-noise $(S / N \geq 200)$ observed spectra. We find that the fraction of the odd isotopes of Ba grows toward the lower Ba abundance (or metallicity) and the mean value in the thick disk stars equals $33 \pm 4 \%$. This indicates the higher contribution of the $r$-process to barium in the thick disk stars compared to the solar system matter. The obtained fraction increases with the [Eu/Ba] abundance ratio growth in agreement with expectations. A significant fraction of the even isotopes of Ba found in old Galactic stars (the thick disk stars), $\sim 67 \%$, is in contrast to the prediction of the "classical" model of the $s$-process and favors the value predicted by the "stellar" models of Arlandini et al. (1999) and Travaglio et al. (1999).
\end{abstract}

Key words. line: formation - nuclear reactions, nucleosynthesis, abundances - stars: abundances - stars: late-type - Galaxy: evolution

\section{Introduction}

The elements heavier than the iron peak are mainly produced through neutron capture reactions in two main processes, the $s$-process (slow) and $r$-process (rapid). As supported by many observational and theoretical results (Travaglio et al. 1999, and references therein), s-nuclei are mainly synthesized during the thermally pulsing asymptotic giant branch phase of low-mass stars $\left(2-4 M_{\odot}\right)$. The $r$-process is associated with explosive conditions in SNeII (see Thielemann et al. 2002, for a general review). Following the pioneering observational study of Spite \& Spite (1978), Truran (1981) has put forward the suggestion that most (if not all) neutron capture elements in very metalpoor stars are of $r$-process origin. Much observational effort was invested in testing this idea (for review, see Truran et al. 2002). To understand the relative importance of the two neutron capture synthesis mechanisms throughout the Galaxy history, stellar element abundance comparisons are made between elements whose solar system isotopic abundances are dominated by the $s$-process and those due mainly to the $r$-process. The europium-to-barium abundance ratio is particularly sensitive to whether nucleosynthesis of the heavy elements occurred by the $s$ - or $r$-process. Other important indicators are the $[\mathrm{Eu} / \mathrm{La}]$ and $[\mathrm{Nd} / \mathrm{Ba}]$ abundance ratios. The most recent determinations of the $[\mathrm{Eu} / \mathrm{Ba}]$ abundance ratios in the samples of metal-poor stars were made by McWilliam (1998), Burris et al. (2000), Fulbright (2000), Mashonkina \& Gehren (2000, 2003),

* Based on observations collected at the German Spanish Astronomical Center, Calar Alto, Spain.
Mishenina \& Kovtyukh (2001) and Bensby et al. (2005). The [Eu/La] abundance ratios in 159 giant and dwarf stars have been measured by Simmerer et al. (2004); the [Nd/Ba] abundance ratios in 46 stars have been derived by Mashonkina et al. (2004). Stellar element abundance ratios have to be compared with theoretical predictions. However, at present, the nuclesynthesis theory is not able to predict the yields of the $r$-process. Based on the hypothesis that the $r$-contributions in the solar system are of primary origin, the $r$-process abundance fractions are obtained by subtracting the $s$-contributions at the epoch of solar formation, estimated with the use of reliable $s$-model, from the solar abundances. One refers to them as $r$-residuals. Two approaches are used to calculate the $s$-process abundance fractions. The "classical" approach relies upon reproducing the product of $n$-capture cross-section and $s$-process abundance (the " $\sigma N$ " curve). "Stellar" $s$-process models are based on nucleosynthesis computations in low- and intermediate-mass AGB stars. The recent "classical" (Arlandini et al. 1999) and "stellar" (Arlandini et al. 1999; Travaglio et al. 1999) models give consistent results within the respective uncertainties for the $s$ process contributions to the most abundant $\mathrm{Ba}$ and $\mathrm{Eu}$ isotopes in the solar system matter, with the only exception being ${ }^{138} \mathrm{Ba}$. Its $r$-residual equals $14 \%$ to $16 \%$ in the "stellar" models and $0 \%$ in the "classical" model. Since ${ }^{138} \mathrm{Ba}$ is the most abundant solar $\mathrm{Ba}$ isotope, the $r$-residual of total $\mathrm{Ba}$ is larger in the "stellar" model by a factor of 2.4 than in the "classical" one. As a result, the "stellar" and "classical" models lead to significantly different values of the solar abundance ratio of $\mathrm{Eu}$ to $\mathrm{Ba}$ contributed by the $r$-process: taken relative 
to the total abundances, $[\mathrm{Eu} / \mathrm{Ba}]_{\mathrm{r}}=0.69$ and $[\mathrm{Eu} / \mathrm{Ba}]_{\mathrm{r}}=1.06$, respectively. The "stellar" model predicts the isotope abundance ratio ${ }^{135} \mathrm{Ba}:{ }^{137} \mathrm{Ba}:{ }^{138} \mathrm{Ba}=26: 20: 54$ in a pure $r$-process nucleosynthesis, while no $\mathrm{Ba}$ even isotope is predicted in the "classical" model.

To reconstruct the evolutionary history of neutron-rich elements in the Galaxy it is, thus, very important to disentangle the fractions of $\mathrm{Ba}$ even and odd isotopes and inspect their abundance as a function of Galactic age. Isotopic shifts of the barium lines are negligible compared to the line width. A determination of the $\mathrm{Ba}$ even-to-odd isotope abundance ratio in stars becomes possible due to the significant hyperfine structure (HFS) affecting the $\mathrm{Ba}$ II resonance lines of the odd isotopes. In total, the resonance line $\lambda 4554$ has 15 components spread over $58 \mathrm{~m} \AA$. The larger the fraction of the odd isotopes, the stronger the HFS broadening of $\lambda 4554$ and the larger the energy absorbed in this line. The total width of the patterns of the Ba II subordinate lines, $\lambda$ 5853, $\lambda 6141$, and $\lambda 6496$, is much smaller, $8 \mathrm{~m} \AA, 8 \mathrm{~m} \AA$, and $23 \AA$, correspondingly.

Cowley \& Frey (1989) pointed out the importance of accounting for HFS in Ba abundance determinations; however, the first attempt to estimate the fraction of the odd isotopes of $\mathrm{Ba}$ in four metal-poor stars was made by Magain \& Zhao (1993a). They used the Ba II subordinate lines, $\lambda 5853$ and $\lambda 6496$, to derive the total $\mathrm{Ba}$ abundance. The $\mathrm{Ba}$ abundance was then deduced from the $\lambda 4554$ line for various isotopic mixtures, the proportion of the odd isotopes being changed until agreement with the subordinate lines was obtained. Magain \& Zhao found an enhancement of the odd isotopes in these stars as compared to solar system matter, in agreement with the expectations. Using the same approach, Mashonkina et al. (1999) did not find significant deviation of the Ba isotopic mixture in the halo star G246-38 $([\mathrm{Fe} / \mathrm{H}]=-2.20)$ from the solar one. In their recent study, Mashonkina et al. (2003) show a distinction between the different Galactic stellar populations with respect to the Ba evento-odd isotope ratio. The halo stars reveal, on average, equal amounts of the odd and even isotopes of $\mathrm{Ba}$; a mean ratio 65:35 $( \pm 10 \%)$ was obtained for the sample of thick disk stars, whereas the solar ratio 82:18 (Anders \& Grevesse 1989) adjusts to observations of the Ba II lines in the sample of thin disk stars.

Magain \& Zhao (1993b) and Magain (1995) have suggested a different method based on measuring the broadening of the Ba II $\lambda 4554$ line. They have applied it to the extremely metalpoor star HD 140283 and found the low fraction of the odd isotopes, $8 \%$, that is consistent with a pure $s$-process production of barium. Using the same method and new high resolution $(R \simeq 200000)$, high signal-to-noise ratio $(S / N \simeq 550)$ spectra of HD 140283, Lambert \& Allende Prieto (2002) draw the opposite conclusion. They find that the $r$-process mixture of the $\mathrm{Ba}$ isotopes predicted by the "stellar" model of Arlandini et al. (1999) provides a fair fit to the observed Ba II $\lambda 4554$ profile.

This study is intended to inspect a history of Ba isotopic fractions in the Galaxy and, thus, a relative importance of the $r$ - and $s$-process in heavy element production through the Galaxy evolution. We determine the abundance ratios of even to odd isotopes of $\mathrm{Ba}$ in the 25 selected stars representing the older (thick disk) and younger (thin disk) Galactic stellar populations. We use the method applied in our previous papers. The results are based on the high resolution $(\sim 60000)$ high signal-to-noise ratio $(S / N \geq 200)$ spectra and non-local thermodynamical equilibrium (NLTE) line formation.

The paper is organized as follows. In Sect. 2 we discuss an accuracy of the used atomic parameters, oscillator strengths and van der Waals damping constants $C_{6}$, and check the investigated $\mathrm{Ba}$ II lines with respect to blending lines. We test our method on solar Ba II lines. Stellar sample, observations, and stellar parameters are described in Sect. 3. In Sect. 4 we present the obtained $\mathrm{Ba}$ even-to-odd isotope ratio in stars and investigate their errors caused by uncertainties of atomic data and stellar parameters. In Sect. 5, stellar fractions of the odd isotopes of $\mathrm{Ba}$ are inspected as indicators of the $r / s$-process nucleosynthesis, and conclusions are given. At the end, the outlook for a determination of $\mathrm{Ba}$ isotopic fractions in very metal-poor stars is estimated.

\section{Method of calculations}

Homogeneous blanketed model atmospheres computed with the MAFAGS code (Fuhrmann et al. 1997) are used in the analysis of both solar and stellar spectra.

\subsection{Atomic parameters}

In the metallicity range of our stellar sample, not only the resonance line, but also one or both of the used subordinate lines either lie on the damping part of the curve of growth or are saturated. In the first turn, our analysis requires very accurate atomic parameters.

As in our previous analysis, in this study we use the absolute oscillator strengths from Reader et al. (1980): $\log g f(\lambda 4554)=$ $0.162, \log g f(\lambda 5853)=-1.0$, and $\log g f(\lambda 6496)=-0.377$. The most recent measurements of Davidson et al. (1992) give $\log g f(\lambda 4554)=0.140, \log g f(\lambda 5853)=-0.91$, and $\log g f(\lambda 6496)=-0.407$. We show below that applying the data of Davidson et al., we cannot agree when fitting parameters of the solar Ba II $\lambda 6496$ and $\lambda 5853$ lines. We do not use the third line of the Ba II $5 d-6 p$ multiplet, $\lambda 6141$ which is blended with the strong Fe I line (Fig. 1).

In our previous studies, we used the data on hyperfine structure components of the Ba II $\lambda 4554$ calculated by Biehl (1976) on the base of Brix \& Kopfermann (1952) measurements. Having reviewed the literature for more recent data, we found that the new fine structure constants of ${ }^{135} \mathrm{Ba}$ and ${ }^{137} \mathrm{Ba}$ obtained by Blatt \& Werth (1982) and Becker \& Werth (1983) lead to a negligible change of the wavelength separations by no more than $1 \%$ for the HFS components of the resonance line. In order to have common input data with other authors that makes a direct comparison of the results possible, in this study all calculations of $\lambda 4554$ are performed using the HFS pattern published by McWilliam (1998). We emphasize that the line profiles computed with the data of Biehl and McWilliam become identical after their relative shift by $0.001 \AA$. Table 1 contains the list of the HFS components of the Ba II $\lambda 4554$. The product of $f_{i j}$ and fractional isotope abundance $\epsilon$ is given for the $\mathrm{Ba}$ isotope mixture in the solar system matter, ${ }^{134} \mathrm{Ba}:{ }^{135} \mathrm{Ba}:{ }^{136} \mathrm{Ba}:{ }^{137} \mathrm{Ba}:{ }^{138} \mathrm{Ba}=$ 2.4:6.6:7.9:11.2:71.8 (Anders \& Grevesse 1989) and that for a pure $r$-process production of $\mathrm{Ba},{ }^{135} \mathrm{Ba}:{ }^{137} \mathrm{Ba}:{ }^{138} \mathrm{Ba}=26: 20: 54$ (Arlandini et al. 1999). Total $f_{i j}=0.727$. The $23 \mathrm{~m} \AA$ wide patterns of the $\lambda 6496$ line are considered as a four component model according to Rutten (1978). Variation in fractional isotope abundances between the solar and pure $r$-process cases has negligible effect on the $\lambda 6496$ line profile, and we use $f \times \epsilon$ computed for the solar mixture of Ba isotopes. We neglect the hyperfine structure of $\lambda 5853$.

The investigated Ba II lines are strongly affected by van der Waals damping. We apply the van der Waals damping constant $\log C_{6}=-31.65$ to $\lambda 4554$. If $\log C_{6}$ is fixed, solar $\mathrm{Ba}$ abundance is immediately determined from the fitting of the solar $\lambda 4554$ line wings. Using the MAFAGS solar model 

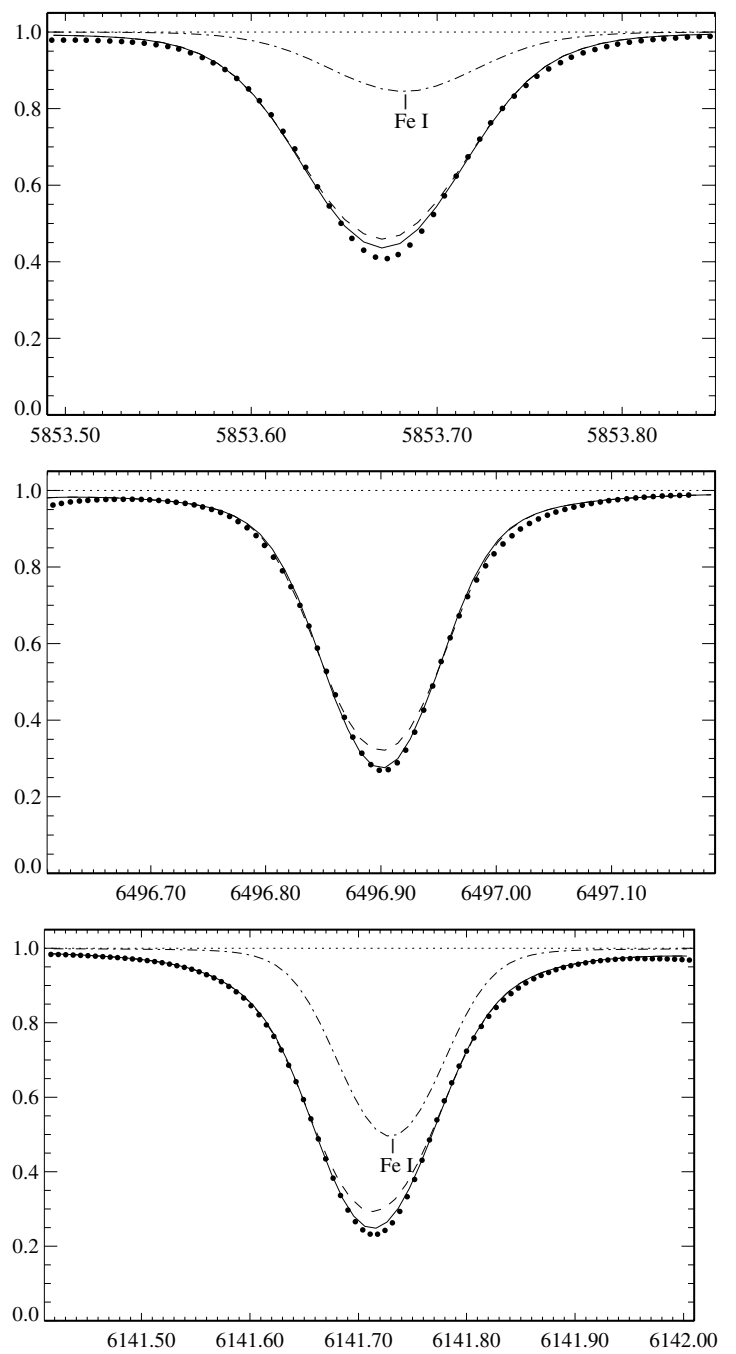

Fig. 1. The best fits of the solar flux Ba II subordinate line profiles (Kurucz et al. 1984, bold dots) achieved using the NLTE approach (continuous line) and LTE assumption (dashed line). Solar Ba abundance $\log \varepsilon_{\mathrm{Ba}}=2.21 ; \log C_{6}=-31.3$. NLTE profiles: each is calculated using $V_{\text {mic }}=0.85 \mathrm{~km} \mathrm{~s}^{-1}$. In the LTE case, $V_{\text {mic }}=0.95 \mathrm{~km} \mathrm{~s}^{-1}(\lambda 5853)$, $1.05 \mathrm{~km} \mathrm{~s}^{-1}\left(\right.$ 6 6496), and $1 \mathrm{~km} \mathrm{~s}^{-1}(\lambda 6141)$. Blending lines are shown in each panel by a dash-dotted line. See text for more details.

atmosphere, we find $\log \varepsilon_{\odot \mathrm{Ba}}=2.21^{1}$. This value agrees within error bars with the meteoritic Ba abundance given by Anders \& Grevesse $\left(1989, \log \varepsilon_{\text {met,Ba }}=2.21 \pm 0.03\right)$ and Grevesse et al. $\left(1996, \log \varepsilon_{\text {met,Ba }}=2.22 \pm 0.02\right)$. Recent determinations by Asplund et al. (2005) lead to the smaller meteoritic $\left(\log \varepsilon_{\mathrm{met}, \mathrm{Ba}}=\right.$ $2.16 \pm 0.03)$ and solar $\left(\log \varepsilon_{\odot \mathrm{Ba}}=2.17 \pm 0.07\right) \mathrm{Ba}$ abundance. For comments, see Note added in proofs.

The Van der Waals damping constant $\log C_{6}$ of the subordinate lines was obtained empirically from the fitting of the solar line profiles under the requirement that unique set of fitting parameters ( $\mathrm{Ba}$ abundance, microturbulence value, and $C_{6}$ value) must adjust observations of the spectral lines belonging to the same multiplet, 5d-6p. Far wings of $\lambda 6496$ impose the upper limit of $\log C_{6}$, provided that solar $\mathrm{Ba}$ abundance is given. We adopt $\log \varepsilon_{\odot \mathrm{Ba}}=2.21$. In this case, $\log C_{6}$ of $\lambda 6496$ cannot be larger than -31.3. Applying this value to $\lambda 5853$ and based on NLTE analysis we obtain a microturbulence value $V_{\text {mic }}=0.85 \mathrm{~km} \mathrm{~s}^{-1}$. The best fit of each line of the multiplet

\footnotetext{
${ }^{1}$ We refer to abundances on the usual scale where $\log \varepsilon_{\mathrm{H}}=12$.
}

Table 1. Atomic data for the HFS components of the Ba II $\lambda$ 4554. I is the relative strength, the product of oscillator strength and fractional isotope abundance, $f \times \epsilon$, is given for two Ba isotope mixtures corresponding to the solar system matter (column "solar") and $r$-process yields predicted by Arlandini et al. (1999).

\begin{tabular}{cccc}
\hline \hline$\lambda, \AA$ & $I$ & \multicolumn{2}{c}{$f \times \epsilon$} \\
\cline { 3 - 4 } & & Solar & $r$-process \\
\hline${ }^{134} \mathrm{Ba}$ & & & \\
4554.032 & 1.000 & 0.0176 & - \\
$135 \mathrm{Ba}$ & & & \\
4554.003 & 0.1562 & 0.0075 & 0.0292 \\
4554.004 & 0.1562 & 0.0075 & 0.0292 \\
4554.005 & 0.0625 & 0.0030 & 0.0117 \\
4554.051 & 0.4375 & 0.0209 & 0.0818 \\
4554.054 & 0.1562 & 0.0075 & 0.0292 \\
4554.055 & 0.0313 & 0.0015 & 0.0058 \\
$136 \mathrm{Ba}$ & & & \\
4554.033 & 1.000 & 0.0568 & - \\
$137 \mathrm{Ba}$ & & & \\
4553.999 & 0.1562 & 0.0129 & 0.0232 \\
4554.001 & 0.1562 & 0.0129 & 0.0232 \\
4554.002 & 0.0625 & 0.0052 & 0.0093 \\
4554.054 & 0.4375 & 0.0360 & 0.0650 \\
4554.056 & 0.1562 & 0.0129 & 0.0232 \\
4554.057 & 0.0313 & 0.0025 & 0.0046 \\
$138 \mathrm{Ba}$ & & & \\
4554.034 & 1.000 & 0.521 & 0.392 \\
\hline
\end{tabular}

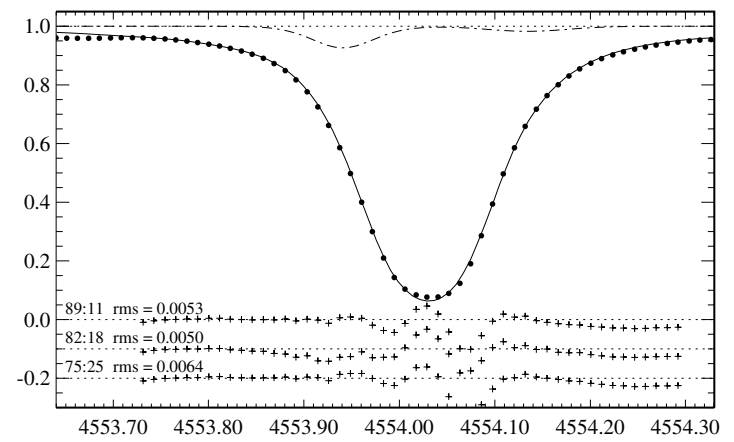

Fig. 2. Synthetic NLTE flux profile of the Ba II resonance line corresponding to the Ba even-to-odd isotope ratio of 82:18 (continuous line) compared with the solar flux profile (bold dots, Kurucz et al. 1984). The differences between observed and calculated spectra, $(\mathrm{O}-\mathrm{C})$, multiplied by a factor of 5, are shown for various even-to-odd isotope ratios in the lower part of figure. Blending lines are shown by a dash-dotted line. See text for more details.

$5 \mathrm{~d}-6 \mathrm{p}$ is achieved at $\log \varepsilon_{\mathrm{Ba}}=2.21, \log C_{6}=-31.3$, and $V_{\text {mic }}=0.85 \mathrm{~km} \mathrm{~s}^{-1}$ (Fig. 1). In Sect. 4 we will discuss an effect of variation in $\log C_{6}$ on $\mathrm{Ba}$ isotope ratio in stars. Analysis of the solar $\lambda 6496$ and $\lambda 5853$ line profiles was also performed with the oscillator strengths from Davidson et al. (1992). Solar $\mathrm{Ba}$ abundance was fixed at $\log \varepsilon_{\odot \mathrm{Ba}}=2.21$. Fitting parameters of two lines turn out to be different: $\lambda 6496$ gives $\log C_{6}=-31.2$ and $V_{\text {mic }}=0.9 \mathrm{~km} \mathrm{~s}^{-1}$, while the significantly lower microturbulence value $V_{\text {mic }}=0.7 \mathrm{~km} \mathrm{~s}^{-1}$ is required to fit the $\lambda 5853$ line profile using $\log C_{6}=-31.2$.

The Ba II $\lambda 4554.034 \AA$ line is blended by the Cr I $\lambda 4553.945$ and $\mathrm{Zr}$ II $\lambda 4553.934$ lines in the blue line wing and by the $\mathrm{CH}$ molecular line at $4554.142 \AA$ in the red line wing (Fig. 2). We have requested the Vienna Atomic Line Data base (VALD, Kupka et al. 1999) and the NIST atomic spectra database (http://physics.nist.gov/PhysRefData) to search for 
atomic parameters of blending atomic lines. The same value $\log g f=-0.73$ based on measurements of Wujec \& Weniger (1981) is given in NIST and VALD for the Cr I $\lambda$ 4553.945. NIST does not contain the $\mathrm{Zr}$ II $\lambda 4553.934$ line, but VALD provides $\log g f=-0.57$, taken from Cowley \& Corliss (1983), for this line. The oscillator strength of the $\mathrm{CH} \lambda 4554.142$ molecular line was fitted to reproduce the observed blend profile; we have obtained $\log g f=-3.2$. The Ba II $\lambda 5853.675 \AA$ line is blended by the Fe I $\lambda 5853.682$ line (Fig. 1). Based on calculations of Kurucz $\&$ Bell (1995), VALD gives $\log g f(\mathrm{Fe}$ I $\lambda$ 5853.682) $=-2.148$. Recently, Kurucz (http : //cfaku5 . cfa . harvard. edu) calculated the new value $\log g f=-2.371$ for this line. We note that the predicted oscillator strength of Fe I $\lambda 5853.682$ has been reduced by a factor of 2.3, compared to the first estimate of Kurucz $\&$ Peytremann (1975), $\log g f=-2.0$. This line is absent in the NIST database. The Ba II $\lambda 6141.713 \AA$ is strongly blended by the Fe I $\lambda 6141.732$ line and not used in further analysis of stellar spectra. With $\log g f(\mathrm{Fe}$ I $\lambda$ 6141) $=-1.61$ taken from the NIST database, we can achieve a good fitting of the solar blend profile (Fig. 1). VALD gives the larger oscillator strength, $\log g f=-1.459$, for Fe I $\lambda 6141$. The contribution of blending lines is treated using the LTE assumption. The chemical abundances are taken from Anders \& Grevesse (1989) in the solar case, and we assume that in the investigated stars, $\mathrm{Cr}$ abundance follows the iron one with $[\mathrm{Cr} / \mathrm{Fe}]=0$, and $\mathrm{Zr}$ abundance follows the barium one with $[\mathrm{Zr} / \mathrm{Ba}]=0$.

\subsection{NLTE line formation}

Our results are based on NLTE line formation for Ba II. The method of NLTE calculations was developed and described earlier (Mashonkina \& Bikmaev 1996; Mashonkina et al. 1999). We use a revised version of the DETAIL program (Butler \& Giddings 1985) based on the accelerated lambda iteration method to solve the coupled radiative transfer and statistical equilibrium equations and the SIU code (wWw.usm.uni-muenchen.de/people/reetz/siu.html) to compute the synthetic line profiles.

In Fig. 1, we present the best fits of the observed Ba II subordinate line profiles in the solar flux atlas of Kurucz et al. (1984) achieved using the NLTE approach and LTE assumption. We emphasize that the unique value of $\mathrm{Ba}$ abundance, $\log \varepsilon_{\mathrm{Ba}}=2.21$, adjusts observations of all these lines. The same value, $\log C_{6}=-31.3$, is used for each line in both the NLTE and LTE calculations. The same microturbulence value, $V_{\text {mic }}=0.85 \mathrm{~km} \mathrm{~s}^{-1}$, is required when the NLTE approach is applied while different values in the LTE case are used: $V_{\text {mic }}=$ $0.95 \mathrm{~km} \mathrm{~s}^{-1}$ for $\lambda 5853, V_{\text {mic }}=1.05 \mathrm{~km} \mathrm{~s}^{-1}$ for $\lambda 6496$, and $V_{\text {mic }}=1 \mathrm{~km} \mathrm{~s}^{-1}$ for $\lambda 6141$. Our synthetic flux profiles are convolved with a profile that combines a rotational broadening of $1.8 \mathrm{~km} \mathrm{~s}^{-1}$ and broadening by macroturbulence with a radialtangential profile. The macroturbulence values $V_{\mathrm{mac}}=3.1 \mathrm{~km} \mathrm{~s}^{-1}$ and $3.0 \mathrm{~km} \mathrm{~s}^{-1}$ are required for $\lambda 5853$ in the NLTE and LTE cases, respectively. The corresponding values for $\lambda 6496$ are $2.3 \mathrm{~km} \mathrm{~s}^{-1}$ and $2.1 \mathrm{~km} \mathrm{~s}^{-1}$ and for $\lambda 6141$, in both cases, $V_{\mathrm{mac}}=2.9 \mathrm{~km} \mathrm{~s}^{-1}$.

\subsection{Can we derive the solar $\mathrm{Ba}$ isotope abundance ratio?}

Assuming the solar system matter $\mathrm{Ba}$ isotopic fractions ${ }^{134} \mathrm{Ba}:{ }^{135} \mathrm{Ba}:{ }^{136} \mathrm{Ba}:{ }^{137} \mathrm{Ba}:{ }^{138} \mathrm{Ba}=2.4: 6.6: 7.9: 11.2: 71.8$, total $\mathrm{Ba}$ abundance $\log \varepsilon_{\mathrm{Ba}}=2.21, \log C_{6}=-31.65$, and using $V_{\text {mic }}=1 \mathrm{~km} \mathrm{~s}^{-1}$, we can achieve a good fitting of the solar $\lambda 4554$ profile. In Fig. 2, we show the best fit obtained by applying $V_{\mathrm{mac}}=3.5 \mathrm{~km} \mathrm{~s}^{-1}$.

The value $V_{\text {mic }}=1 \mathrm{~km} \mathrm{~s}^{-1}$ obtained from the resonance line does not agree with the value $V_{\text {mic }}=0.85 \mathrm{~km} \mathrm{~s}^{-1}$ found from the subordinate lines. We are aware that a phenomenological description of the velocity field using a microturbulence value is a rough approximation and that it fails to treat spectral lines formed over a wide range of depths. The $\lambda 4554$ line core is formed in very outer layers near $\log \tau_{5000}=-4.7$, while radiation of the subordinate lines comes from the deeper layers, below $\log \tau_{5000}=-2.8$ for $\lambda 6496$ and below $\log \tau_{5000}=-1.9$ for $\lambda 5853$. We have tried to answer the question of whether we are able to derive a solar atmosphere $\mathrm{Ba}$ isotope abundance ratio from the HFS affecting the $\lambda 4554$ line profile without a reference to the subordinate lines. In addition to the even-to-odd isotope ratio $82: 18$ corresponding to solar system matter, a fitting of the solar $\lambda 4554$ line profile was made assuming the ratios $89: 11$ and 75:25. We suppose that the proportion ${ }^{135} \mathrm{Ba}:{ }^{137} \mathrm{Ba}$ does not change. It is not the case if the $r / s$-process contribution to Ba varies. However, as can be seen from Table 1, the $\lambda 4554$ line profile depends on the total fractional abundance of the odd isotopes rather than on the proportion ${ }^{135} \mathrm{Ba}:{ }^{137} \mathrm{Ba}$. Our test computations for the isotope mixture ${ }^{135} \mathrm{Ba}:{ }^{137} \mathrm{Ba}:\left({ }^{134} \mathrm{Ba}+\right.$ $\left.{ }^{136} \mathrm{Ba}+{ }^{138} \mathrm{Ba}\right)=2: 9: 89$ predicted by Arlandini et al. (1999) for a pure $s$-process production of Ba give the $\lambda 4554$ line profile fully consistent with that for the ratio ${ }^{135} \mathrm{Ba}:{ }^{137} \mathrm{Ba}:\left({ }^{134} \mathrm{Ba}+\right.$ $\left.{ }^{136} \mathrm{Ba}+{ }^{138} \mathrm{Ba}\right)=5.5: 5.5: 89$. In calculations with various evento-odd isotope ratio, a microturbulence value was a free parameter, and in each case the best fit was found from the minimum root mean square (rms) difference between observed and calculated spectra. $V_{\mathrm{mac}}$ was fixed at $3.5 \mathrm{~km} \mathrm{~s}^{-1}$. The minimum rms is achieved at $V_{\text {mic }}=1.15 \mathrm{~km} \mathrm{~s}^{-1}$ for the even-to-odd isotope ratio 89:11, and $V_{\text {mic }}=0.9 \mathrm{~km} \mathrm{~s}^{-1}$ for 75:25. In the latter case, a shift of the observed profile by $-0.0008 \AA$ is required. Figure 2 shows the obtained differences between observed and calculated spectra, $(\mathrm{O}-\mathrm{C})$, multiplied by a factor of 5 , for various isotope ratios.

The rms values quoted in Fig. 2 are very similar for various isotope mixtures. The obtained $V_{\text {mic }}$ values $0.9 \mathrm{~km} \mathrm{~s}^{-1}$ to $1.15 \mathrm{~km} \mathrm{~s}^{-1}$ are within the interval of widely used solar microturbulence values, and no value can be preferred. The $(\mathrm{O}-\mathrm{C})$ values are the largest in the line core within $\pm 0.07 \AA$ from the line center, independent of isotope ratio. The $\lambda 4554$ line core is most probably influenced by a non-thermal and depth-dependent chromospheric velocity field that is not part of our solar model. We note that the chromospheric temperature rise, as well as the horizontal temperature inhomogeneity seen as solar granulation, is expected to be less important because $\mathrm{Ba}$ II is the dominant ionization stage.

We conclude from these results that a precise determination of the proportion of the even and odd isotopes of $\mathrm{Ba}$ in the solar atmosphere matter requires a correct treatment of the atmospheric velocity field that is only possible on the basis of hydrodynamic calculations. In metal-poor stars, the $\lambda 4554$ line forms in the deeper atmospheric layers compared to the Sun and in smaller extension is expected to be affected by hydrodynamic phenomena of the very surface layers.

\section{Stellar sample, observations, and stellar parameters}

All the 25 stars were selected from Fuhrmann's (2004) list. Each star was observed with a resolution of $\sim 60000$, at least 

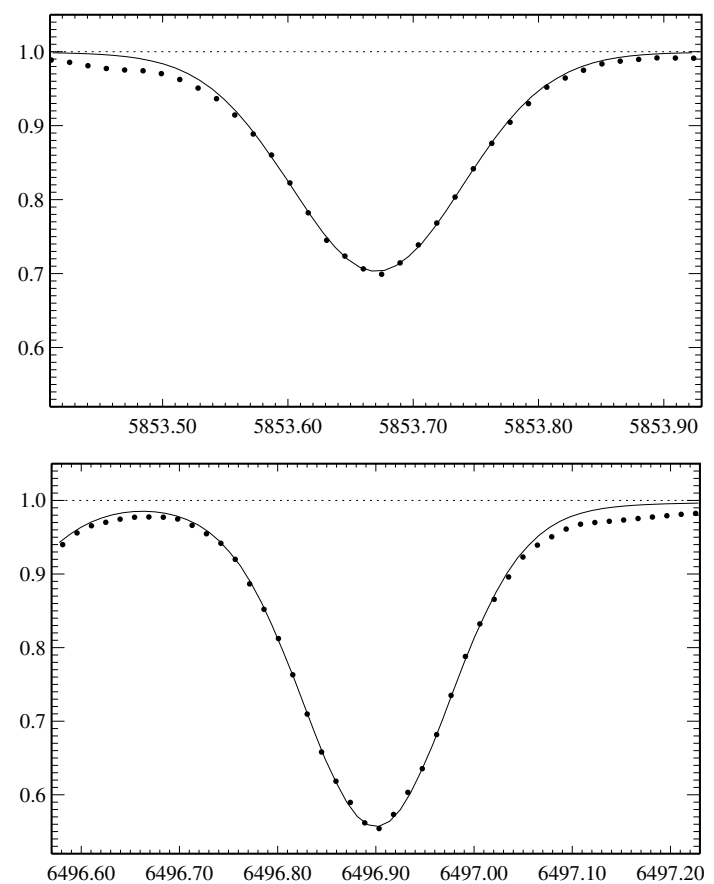

Fig. 3. Synthetic NLTE (continuous line) flux profiles of the Ba II subordinate lines compared with the observed FOCES spectra (bold dots) of $\mathrm{HD} 10519$. The same value $[\mathrm{Ba} / \mathrm{H}]=-0.69$ is obtained from both lines.

twice, and for each star both the Ba II subordinate lines, $\lambda 5853$ and $\lambda 6496$, are available in its spectra. Spectra were obtained by Klaus Fuhrmann using the fiber optics Cassegrain echelle spectrograph FOCES at the $2.2 \mathrm{~m}$ telescope of the Calar Alto Observatory in 1997-2000. The signal-to-noise ratio is 200 or higher in the spectral range $\lambda>4500 \AA$. Typical line profiles are seen in Figs. 3 and 5.

The list of stars under investigation is given in Table 2 . Stellar parameters have been determined spectroscopically by Fuhrmann (2004). Their errors are estimated as $\Delta T_{\text {eff }}=80 \mathrm{~K}$, $\Delta \log g=0.1, \Delta[\mathrm{Fe} / \mathrm{H}]=0.1 \mathrm{dex}$, and $\Delta V_{\text {mic }}=0.2 \mathrm{~km} \mathrm{~s}^{-1}$. We use also his identification of a membership of individual stars in the particular stellar population that is based on the star's kinematics, metallicity, $\alpha$-enhancement, and age. Our sample includes 15 thick disk stars, 9 thin disk stars, and one halo star.

\section{Results}

\subsection{Stellar total Ba abundances}

Ba abundances were derived from the Ba II $\lambda 5853$ and $\lambda 6496$ line profile fitting, and then the average value was calculated. The $[\mathrm{Ba} / \mathrm{H}]$ values calculated using solar $\mathrm{Ba}$ abundance $\log \varepsilon_{\mathrm{Ba}}=2.21$ are presented in Table 2 . We note that in this paper we revise our earlier determinations (Mashonkina \& Gehren 2001) of Ba abundance in the investigated stars. Contrary to the previous analysis, we use the same value $\log C_{6}$ for both subordinate lines and treat the $\lambda 5853$ blend taking into account both the $\mathrm{Ba}$ II and Fe I lines (see Sect. 2.1). A reduction of the van der Waals damping constant from $\log C_{6}=-30.6$ for $\lambda 5853$ and $\log C_{6}=-31.2$ for $\lambda 6496$ used in 2001 to our present estimate $\log C_{6}=-31.3$ (Sect. 2.1) leads to an increase of derived $\mathrm{Ba}$ abundance, while accounting for absorption by the blending of the Fe I line leads to a decrease of Ba abundance from the $\lambda 5853$ blend. As a result, in the two most metal-poor stars of our sample (HD 103095 and HD 64606), the revised $\mathrm{Ba}$ abundance is smaller (van der Waals broadening of the
Table 2. Stellar parameters, Ba abundances, and the fractions of the odd isotopes of Ba (column odd, in \%) of the selected sample. The column low-up contains the lower and upper limits of that fraction. In the column "Note", the notations 0,1 , and 2 refer to the thin disk, thick disk, and halo stars, respectively. $V_{\text {mic }}$ is given in $\mathrm{km} \mathrm{s}^{-1}$.

\begin{tabular}{rrrrrrrrr}
\hline \hline HD & $T_{\text {eff }}$ & $\log g$ & $V_{\text {mic }}$ & {$[\mathrm{Fe} / \mathrm{H}]$} & {$[\mathrm{Ba} / \mathrm{H}]$} & odd & low-up & Note \\
\hline 3795 & 5370 & 3.82 & 1.0 & -0.64 & -0.58 & 39 & $34-44$ & 1 \\
4614 & 5940 & 4.33 & 1.0 & -0.30 & -0.23 & 24 & $17-31$ & 0 \\
9407 & 5660 & 4.42 & 0.9 & 0.03 & -0.01 & 13 & $7-18$ & 0 \\
10519 & 5710 & 4.00 & 1.1 & -0.64 & -0.69 & 42 & $38-46$ & 1 \\
10697 & 5610 & 3.96 & 1.0 & 0.10 & 0.14 & 11 & $4-17$ & 0 \\
18757 & 5710 & 4.34 & 1.0 & -0.28 & -0.38 & 31 & $26-36$ & 1 \\
22879 & 5870 & 4.27 & 1.2 & -0.86 & -0.88 & 31 & $28-34$ & 1 \\
30649 & 5820 & 4.28 & 1.2 & -0.47 & -0.57 & 30 & $27-33$ & 1 \\
37124 & 5610 & 4.44 & 0.9 & -0.44 & -0.55 & 29 & $25-34$ & 1 \\
52711 & 5890 & 4.31 & 1.0 & -0.16 & -0.09 & 16 & $11-22$ & 0 \\
55575 & 5890 & 4.25 & 1.0 & -0.36 & -0.39 & 23 & $20-26$ & 0 \\
62301 & 5940 & 4.18 & 1.2 & -0.69 & -0.75 & 32 & $28-36$ & 1 \\
64606 & 5320 & 4.54 & 1.0 & -0.89 & -1.00 & 35 & $30-40$ & 1 \\
65583 & 5320 & 4.55 & 0.8 & -0.73 & -0.77 & 39 & $34-44$ & 1 \\
68017 & 5630 & 4.45 & 0.9 & -0.40 & -0.50 & 33 & $25-38$ & 1 \\
69611 & 5820 & 4.18 & 1.2 & -0.60 & -0.72 & 28 & $24-32$ & 1 \\
102158 & 5760 & 4.24 & 1.1 & -0.46 & -0.59 & 35 & $29-43$ & 1 \\
103095 & 5110 & 4.66 & 0.8 & -1.35 & -1.41 & 42 & $36-46$ & 2 \\
112758 & 5240 & 4.62 & 0.7 & -0.43 & -0.53 & 27 & $16-37$ & 1 \\
114762 & 5930 & 4.11 & 1.2 & -0.71 & -0.83 & 37 & $31-42$ & 1 \\
117176 & 5480 & 3.83 & 1.0 & -0.11 & -0.09 & 25 & $11-38$ & 0 \\
121560 & 6140 & 4.27 & 1.2 & -0.43 & -0.35 & 27 & $22-32$ & 0 \\
132142 & 5240 & 4.58 & 0.7 & -0.39 & -0.46 & 33 & $29-37$ & 1 \\
134987 & 5740 & 4.25 & 1.0 & 0.25 & 0.17 & 18 & $13-21$ & 0 \\
168009 & 5785 & 4.23 & 1.0 & -0.03 & -0.07 & 16 & $12-21$ & 0 \\
\hline
\end{tabular}

subordinate lines is weak and blending of Ba II $\lambda 5853$ prevails) by 0.01 dex compared to our previous data. Also in the remaining stars, the revised $\mathrm{Ba}$ abundance has become larger by $0.02-$ 0.04 dex, with the only exception being that HD 117176 now reveals $\mathrm{Ba}$ abundance that is higher by 0.06 dex. The latter star shows the largest discrepancy in $\log \varepsilon_{\mathrm{Ba}}$ derived from the $\lambda 5853$ and $\lambda 6496$ lines.

Ba abundance determined from the $\lambda 5853$ line turns out to be systematically lower than that from the second line; the $\mathrm{Ba}$ abundance is lower by $-0.03 \pm 0.02 \mathrm{dex}$ in the stars with $[\mathrm{Ba} / \mathrm{H}] \geq-0.35$, by $-0.01 \pm 0.03$ dex in the more Ba-poor stars, and by $-0.02 \pm 0.03$ dex for the whole sample (Fig. 4, top panel). The abundance difference $\log \varepsilon(\lambda 5853)-\log \varepsilon(\lambda 6496)$ shows no correlation with $T_{\text {eff }}$ (Fig. 4 , bottom panel). Ba abundance from the $\lambda 5853$ line can be underestimated due to overestimation of the oscillator strength of the blending Fe I $\lambda 5853$ line. Using $\log g f=-2.371$ calculated recently by Kurucz (http://cfaku5. cfa.harvard.edu), we derive the larger Ba abundance from the $\lambda 5853$ blend: it is larger by 0.01 dex in $\mathrm{HD} 9407([\mathrm{Ba} / \mathrm{H}]=-0.01)$ and by $0.02 \mathrm{dex}$ in $\mathrm{HD} 68017$ $([\mathrm{Ba} / \mathrm{H}]=-0.50)$ and HD $64606([\mathrm{Ba} / \mathrm{H}]=-1.00)$. Another source of the obtained discrepancy between the two Ba II lines is the uncertainty of the van der Waals damping constant of 0.050.18 dex according to Barklem (2006). In the next subsection, we will discuss an effect of total $\mathrm{Ba}$ abundance error on the $\mathrm{Ba}$ even-to-odd isotope ratio in stars.

\subsection{Ba even-to-odd isotope ratio in stars}

The fraction of the odd isotopes of $\mathrm{Ba}$ is derived from the requirement that $\mathrm{Ba}$ abundances derived from the $\mathrm{Ba}$ II resonance 

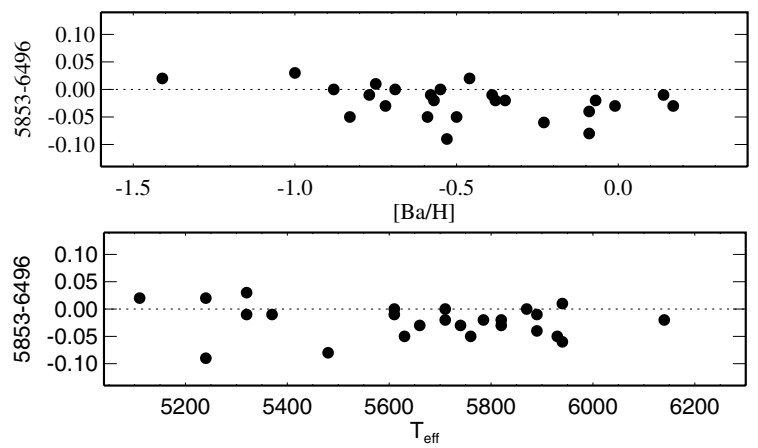

Fig. 4. NLTE abundance differences $\log \varepsilon(\lambda 5853)-\log \varepsilon(\lambda 6496)$ versus total $\mathrm{Ba}$ abundance $[\mathrm{Ba} / \mathrm{H}]$ (top panel) and $T_{\text {eff }}$ (bottom panel).

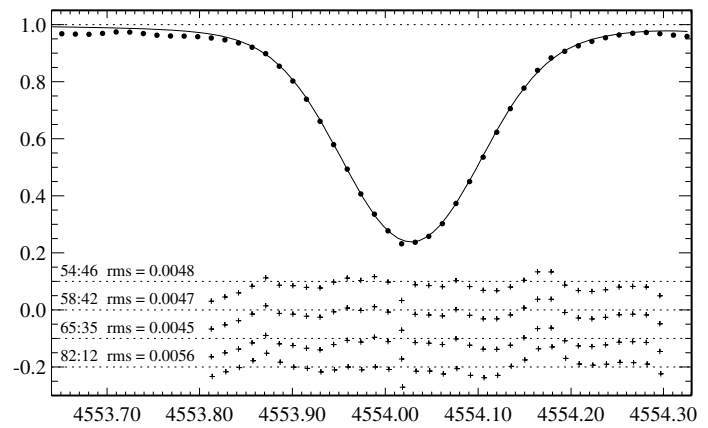

Fig. 5. Synthetic NLTE flux profile corresponding to $\log \varepsilon_{\mathrm{Ba}}=1.52$ and $\mathrm{Ba}$ even-to-odd isotope ratio 58:42 (continuous line) compared to the observed profile (bold dots) of the Ba II resonance line in HD 10519. $\log \varepsilon_{\mathrm{Ba}}=1.51$ for the isotope ratio $54: 46, \log \varepsilon_{\mathrm{Ba}}=1.54$ for $65: 35$, and $\log \varepsilon_{\mathrm{Ba}}=1.64$ for $82: 18$. The $(\mathrm{O}-\mathrm{C})$ values multiplied by a factor of 5 are shown for various isotope mixtures in the lower part of the figure. See text for more details.

line and the subordinate lines must be equal. We emphasize that absolute $\mathrm{Ba}$ abundance $\log \varepsilon_{\mathrm{Ba}}$ is used in this procedure, and, thus, the obtained fraction does not depend on the adopted value of solar Ba abundance.

Figure 5 illustrates a determination procedure for one of the stars of our sample, HD 10519. The same Ba abundance, $\log \varepsilon_{\mathrm{Ba}}=1.52$, is obtained from both subordinate lines. The $\lambda 4554$ line gives $\log \varepsilon_{\mathrm{Ba}}=1.64$ at the assumption of the isotope mixture in solar system matter and $\log \varepsilon_{\mathrm{Ba}}=1.51$ for the pure $r$-process yields predicted by Arlandini et al. (1999). As noted above, the synthetic $\lambda 4554$ line profile depends on the total fractional abundance of the odd isotopes rather than on the exact abundance of each odd isotope. Therefore, we vary the even-to-odd isotope ratio (from here on, "the isotope ratio") in calculations of $\lambda 4554$ until the obtained $\mathrm{Ba}$ abundance agrees with that from the subordinate lines. The required $\mathrm{Ba}$ abundance $\log \varepsilon_{\mathrm{Ba}}=1.52$ is achieved from $\lambda 4554$ when the isotope ratio equals 58:42. The following points should be noted.

- When we go from the isotope ratio 82:18 to 54:46, the relative shift of the observed profile by $0.001 \AA$ is required to achieve the best fit.

- We define the best fit from a requirement of the minimum rms difference between observed and calculated spectra. The equivalent width corresponding to the best fit may be different for different isotope ratios, e.g., in our calculations for HD 10519, the equivalent width measured over the same spectral interval, 4553.8-4554.3 $\AA, \mathrm{W}(\lambda 4554)=154.1 \mathrm{~m} \AA$ for the isotope ratio $82: 18$ and $152.2 \mathrm{~m} \AA$ for $54: 46$. This can be understood. The smaller fraction of the odd isotopes that
Table 3. Effect on the Ba odd isotope fraction (in \%) caused by uncertainties of atomic data and stellar parameters.

\begin{tabular}{llccc}
\hline \hline Input & Input & \multicolumn{3}{c}{$[\mathrm{Ba} / \mathrm{H}]$} \\
\cline { 3 - 5 } parameter & error & -0.01 & -0.50 & -1.00 \\
\hline $\log C_{6}(5 d-6 p)$ & +0.1 & +6 & +3.8 & +1.4 \\
$\log \varepsilon_{\mathrm{Ba}}$ & -0.02 & +4 & +5 & +5.6 \\
$T_{\text {eff }}(\mathrm{K})$ & +80 & +3 & +4 & +4 \\
$\log g$ & -0.1 & $<1$ & -1 & +3 \\
$V_{\text {mic }}\left(\mathrm{km} \mathrm{s}^{-1}\right)$ & +0.1 & +5 & $<1$ & $<1$ \\
$\log g f(\lambda 4554)$ & -0.02 & +4 & +5 & +5.6 \\
$\log C_{6}(\lambda 4554)$ & +0.1 & -7 & -8 & -8 \\
\hline
\end{tabular}

the larger Ba abundance is required to fit, in the observed line profile, and the calculated line wings, which never perfectly fit the observed ones (Fig. 5), contribute to $\mathrm{W}_{\lambda}$ in the greater extension, when compared to the case of the larger fraction of the odd isotopes with the smaller $\mathrm{Ba}$ abundance.

- The macroturbulence value was allowed to be free because we have no arguments to fix it. An increase of the fraction of the odd isotopes cancels, in part, a saturation of $\lambda 4554$, and the line formation depth shifts downward; e.g., in our calculations for HD 10519, a macroturbulence value grows from $4.25 \mathrm{~km} \mathrm{~s}^{-1}$ to $4.5 \mathrm{~km} \mathrm{~s}^{-1}$ when the isotope ratio changes from $82: 18$ to $54: 46$.

Uncertainty of the fractional abundance of the odd isotopes of $\mathrm{Ba}$ in stars. Random errors of a desirable fraction are due to total $\mathrm{Ba}$ abundance error and the uncertainty of stellar parameters, $T_{\text {eff }}, \log g$, and microturbulence value. Systematical errors are caused by the uncertainty of the Ba II $\lambda 4554$ atomic parameters, namely, $f_{i j}$ and $C_{6}$ values. Varying all mentioned parameters, we have performed the test calculations for the three representative stars of our sample with different values of $[\mathrm{Ba} / \mathrm{H}], \mathrm{HD} 9407$ $([\mathrm{Ba} / \mathrm{H}]=-0.01), \mathrm{HD} 68017([\mathrm{Ba} / \mathrm{H}]=-0.50)$, and HD 64606 $([\mathrm{Ba} / \mathrm{H}]=-1.00)$. The effect on the $\mathrm{Ba}$ odd isotope fraction in these stars is shown in Table 3.

The most important source of total $\mathrm{Ba}$ abundance error in the stars with metallicity close to solar one is the uncertainty of the van der Waals damping constant of the subordinate lines. A variation in $\log C_{6}$ of 0.1 translates to the 0.03 dex variation in $\log \varepsilon_{\mathrm{Ba}}$. When $\lambda 4554$ is saturated, a reduction of $\mathrm{Ba}$ abundance obtained from the subordinate lines by 0.01 dex requires an increase of the fractional abundance of the odd isotopes by $\sim 2 \%$ to $\sim 3 \%$ depending on the resonance line strength. A variation of $80 \mathrm{~K}$ in $T_{\text {eff }}$ translates to the uncertainty of the obtained fraction of $3 \%$ to $4 \%$. In the stars with $[\mathrm{Ba} / \mathrm{H}] \leq-0.5$, a variation in $V_{\text {mic }}$ has nearly the same effect on both the resonance and subordinate lines, and no change of the $\mathrm{Ba}$ odd isotope fraction is required. In the stars with metallicity close to solar one, the resonance line lies on the damping part of the curve of growth and is weakly sensitive to $V_{\text {mic }}$ variations, while this is not the case for the subordinate lines. The uncertainties of total Ba abundance, $T_{\text {eff }}, \log g$, and $V_{\text {mic }}$, in total, result in a random error of the obtained fraction of $7 \%, 9 \%$, and $10 \%$ in the stars with $[\mathrm{Ba} / \mathrm{H}]=0,-0.5$, and -1 , correspondingly. There can be also a systematical difference of $4 \%$ to $5 \%$ between the thick disk and thin disk stars, due to a discrepancy in the mean value $\log \varepsilon_{\mathrm{Ba}}(\lambda 5853)-\log \varepsilon_{\mathrm{Ba}}(\lambda 6496)$ between the samples of thick disk and thin disk stars.

A variation of 0.1 dex in the van der Waals damping constant of the $\lambda 4554$ line produces a strong effect, $7 \%$ to $8 \%$ of the derived fraction of the odd isotopes of $\mathrm{Ba}$; however, this is a systematical effect. It depends only weakly on $\mathrm{Ba} / \mathrm{H}$ and 
therefore will not affect a difference in the fraction of the odd isotopes between different stars.

We suppose that in each individual star, the spread of $\mathrm{Ba}$ abundance derived from two subordinate lines fully reflects both random and systematical errors of total $\mathrm{Ba}$ abundance, including effects of the uncertainties of atomic parameters $\left(g f\right.$ and $C_{6}$ values), microturbulence value, and NLTE treatment. It is worth while to remember that due to different strengths of the Ba II $\lambda 6496$ and $\lambda 5853$ lines $(f(\lambda 6496) / f(\lambda 5853) \simeq 4)$, they lie in each star on different parts of the curve of growth. The uncertainty of a desirable fraction in each star is evaluated, taking into account its individual $\mathrm{Ba}$ abundance error and uncertainty of the $\lambda 4554$ line profile fitting. In terms of $\mathrm{Ba}$ abundance, the latter value is estimated to be $0.01 \mathrm{dex}$, based on the analysis of the (O-C) values. For example, in HD 10519, both subordinate lines give the same $\log \varepsilon_{\mathrm{Ba}}$ and the lower and upper limits of the odd isotope fraction are calculated taking into account only the uncertainty of the $\lambda 4554$ line profile fitting $(0.01 \mathrm{dex})$. In the range of the odd isotope fraction between $35 \%$ and $46 \%$, characteristic of this star, a $\mathrm{Ba}$ abundance variation of 0.01 dex produces the $4 \%$ variation in the fractional abundance of the odd isotopes. Therefore, our estimate of the fraction of the odd isotopes of $\mathrm{Ba}$ in this star is $42 \pm 4 \%$.

The obtained fraction of the odd isotopes, with its lower and upper limits in the stars of our sample, are presented in Table 2.

\section{Stellar Ba odd isotopes as an indicator of the $\mathrm{r} / \mathrm{s}$-process nucleosynthesis}

In Fig. 6, we plot the fractional abundance of the odd isotopes of $\mathrm{Ba}$ versus the total $\mathrm{Ba}$ abundance and the $[\mathrm{Eu} / \mathrm{Ba}]$ and $[\mathrm{Eu} / \mathrm{Fe}]$ abundance ratios. Based on the spread of $\mathrm{Ba}$ abundance in each star (Fig. 4), error bars of the $[\mathrm{Ba} / \mathrm{H}]$ value do not exceed 0.1 dex. The $[\mathrm{Eu} / \mathrm{Fe}]$ abundance ratios are taken from our earlier determinations (Mashonkina \& Gehren 2001), where their errors are estimated as $\Delta[\mathrm{Eu} / \mathrm{Fe}]=0.05$ dex. We note that both $\mathrm{Eu}$ and $\mathrm{Fe}$ abundances are derived from weak spectral lines of the dominant ionization stage, Eu II and Fe II, respectively, and the uncertainty of stellar parameters has a negligible effect on the derived $\mathrm{Eu} / \mathrm{Fe}$ abundance ratio. Error bars of the $[\mathrm{Eu} / \mathrm{Ba}]$ value are mainly defined by errors of $\mathrm{Ba}$ abundance.

It is clearly seen that the obtained fraction grows towards the lower $\mathrm{Ba}$ abundance. Its value in the thick disk stars ranges between $27 \%$ and $42 \%$, with the mean value $33 \pm 4 \%$. This indicates the higher contribution of the $r$-process to barium in the thick disk stars compared to the solar system matter in agreement with our expectations. Analysis of the $[\alpha / \mathrm{Fe}]$ abundance ratios (we cite here only the first such studies: Gratton et al. 1996, 2000; Fuhrmann 1998; Prochaska et al. 2000) suggests that the thick disk stellar population is nearly the same age as the Galactic halo one. This conclusion was confirmed from an analysis of $\mathrm{Eu} / \mathrm{Ba}$ and $\mathrm{Nd} / \mathrm{Ba}$ abundance ratios (Mashonkina \& Gehren 2000; Mashonkina et al. 2004; Bensby et al. 2005). The significant overabundance of $\mathrm{Eu}$ and $\mathrm{Nd}$ relative to $\mathrm{Ba}$ found in the thick disk stars at $[\mathrm{Fe} / \mathrm{H}] \leq-0.3$ says that these stars formed in the early Galaxy when SNeII dominated the synthesis of heavy elements.

Both the $[\mathrm{Eu} / \mathrm{Ba}]$ abundance ratio and the fractional abundance of the odd isotopes of $\mathrm{Ba}$ are sensitive to the relative contribution of the $r$-process to heavy element synthesis, and it is natural to expect their correlation. Figure 6 shows that such correlation really exists. It is more clearly seen in the plane fractional abundance of the odd isotopes of $\mathrm{Ba}-[\mathrm{Eu} / \mathrm{Fe}]$ because the
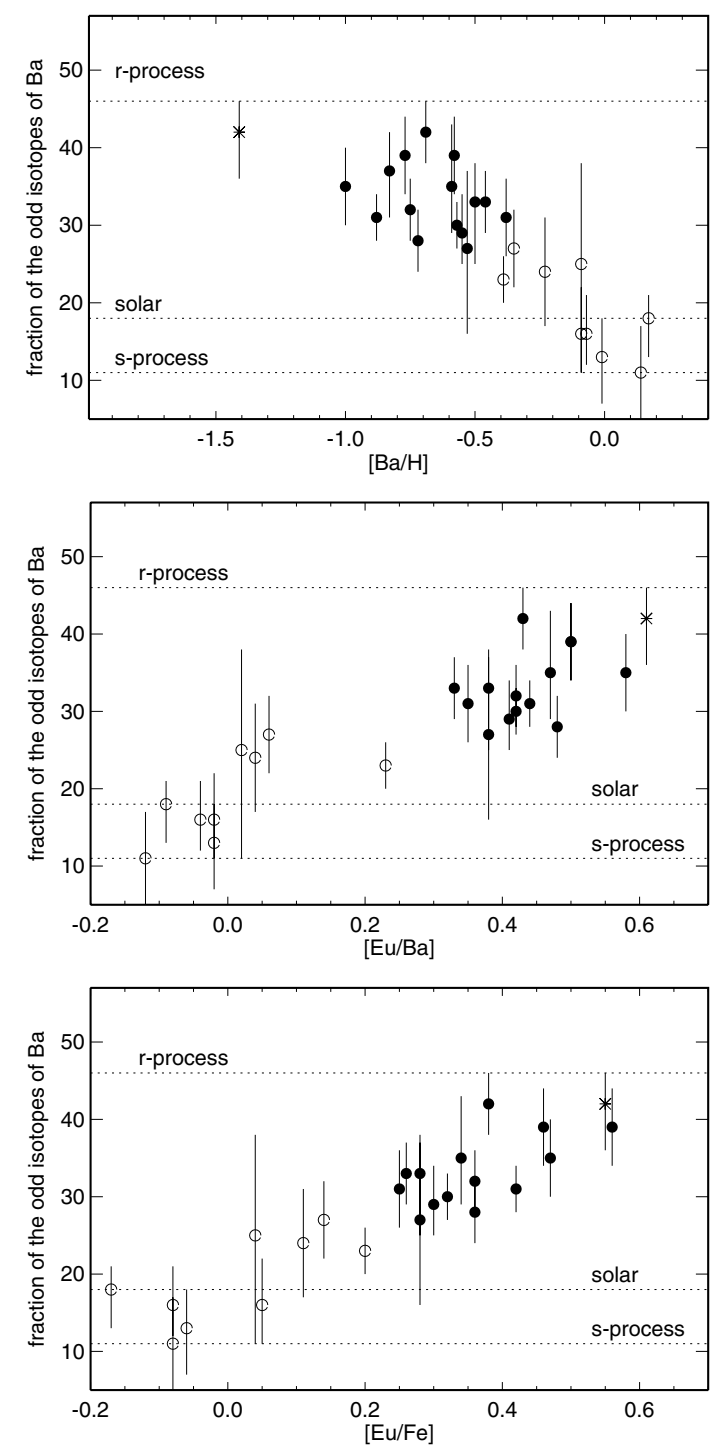

Fig. 6. The fractional abundance of the odd isotopes of Ba versus $[\mathrm{Ba} / \mathrm{H}]$ (top panel), [Eu/Ba] (middle panel), and $[\mathrm{Eu} / \mathrm{Fe}]$ (bottom panel). The uncertainty of individual values is shown by vertical lines. Horizontal lines indicate the solar fraction of the odd isotopes, $18 \%$, and the values predicted by Arlandini et al. (1999) and Travaglio et al. (1999) for a pure $r$-process, $46 \%$, and pure $s$-process, $11 \%$, production of barium.

range of $[\mathrm{Eu} / \mathrm{Fe}]$ values in the thick disk stars is larger than that of $[\mathrm{Eu} / \mathrm{Ba}]$, and errors of the $[\mathrm{Eu} / \mathrm{Fe}]$ values are smaller than that of $[\mathrm{Eu} / \mathrm{Ba}]$.

The obtained data provide observational constraints to models of the $s$ - and $r$-process nucleosynthesis. The "classical" model of the $s$-process based on the newest accurate measurements of the stellar $n$-capture cross-sections (Arlandini et al. 1999) predicts a pure $s$-process origin of solar even isotopes of $\mathrm{Ba}$. In this model, no even isotope of $\mathrm{Ba}$ appears in stars formed in the early Galaxy before the onset of the $s$-process in AGB stars. In contrast to this prediction, we find a significant fraction of the even isotopes of $\mathrm{Ba}, \sim 67 \%$, in old Galactic stars, the thick disk stars. The "stellar" models of Arlandini et al. (1999), based on stellar AGB models of $1.5 M_{\odot}$ and $3 M_{\odot}$ with half solar metal abundance, and Travaglio et al. (1999), who integrate $s$-abundances from different generations of AGB stars, i.e., consider the whole range of Galactic metallicities, both predict that solar abundance of isotope ${ }^{138} \mathrm{Ba}$ is contributed to 
by the $s$-process only in part: $86 \%$ and $84 \%$, respectively. As a result, in the oldest stars of the Galaxy, formed at the epoch of SNeII dominance in nucleosynthesis, a fraction of the even isotopes of $\mathrm{Ba}$ is expected to be at the level of $54 \%$. Ba isotopic fractions found in the thick disk stars favor the "stellar" model of heavy element synthesis.

Note added in proof: For $\lambda 4554$, the van der Waals damping constant based on theoretical predictions of Barklem \& O'Mara (1998) is equal to $\log C_{6}=-31.46$ with the uncertainty of $0.05-$ 0.18 dex according to Barklem \& Aspelund-Johansson (2005) and Barklem (2006). Using this value and the MAFAGS solar model atmosphere, we find solar $\mathrm{Ba}$ abundance $\log \varepsilon_{\odot \mathrm{Ba}}=$ 2.17, in well agreement with the recent determinations of the meteoritic $\left(\log \varepsilon_{\text {met,Ba }}=2.16 \pm 0.03\right)$ and solar $\left(\log \varepsilon_{\odot \mathrm{Ba}}=\right.$ $2.17 \pm 0.07) \mathrm{Ba}$ abundance by Asplund et al. (2005). Assuming $\log C_{6}=-31.46$ for $\lambda 4554$, we checked other stars of our sample with stellar parameters close to solar ones, HD 9407, HD 10697 , HD 134987, and HD 168009 (see Sect. 3), and found systematically lower $\mathrm{Ba}$ abundance from the $\mathrm{Ba}$ II resonance line compared to that from the subordinate lines, by 0.06 dex to 0.10 dex. Variation of stellar parameters and microturbulence value does not help to achieve agreement between different lines, and the only way out is to reduce collisional broadening of the resonance line. Therefore, we apply in this study the value $\log C_{6}=-31.65$ to $\lambda 4554$. For the Ba II multiplet $5 d-6 p$, the van der Waals damping constant $\log C_{6}=-31.3$ found empirically in Sect. 2.1 is very close to $\log C_{6}=-31.28$ based on the theory of Barklem \& O'Mara (1998) and accessible via the Vienna Atomic Line Data base (VALD, Kupka et al. 1999).

Acknowledgements. We are very grateful to Klaus Fuhrmann for providing reduced stellar spectra and Thomas Gehren for providing a Windows version of the code DETAIL. ML acknowledges with gratitude the National Astronomical Observatories of Chinese Academy of Science for warm hospitality during a productive stay in October-December of 2005. We thank the anonymous referee for useful remarks and comments. This research was supported by the Deutsche Forschungsgemeinschaft with grant 436 RUS 17, the Russian Foundation for Basic Research with grant 05-02-39005-GFEN-a, the Natural Science Foundation of China with grants NSFC 10433010 and 10521001, the RF President with a grant on Leading Scientific Schools 1789.2003.2, and the Presidium RAS Programme "Origin and evolution of stars and the Galaxy".

\section{Appendix A: The outlook for a determination of $\mathrm{Ba}$ isotopic fractions in very metal-poor stars}

It would be very important to extend our study to the older and more metal-poor stars revealing a pure $r$-process nucleosynthesis and to determine from observations the relative yields of the even and odd isotopes of $\mathrm{Ba}$ in the $r$-process. At $[\mathrm{Ba} / \mathrm{H}]<-2$, our method fails to give a reliable value of the fractional abundance of the odd isotopes of $\mathrm{Ba}$ due to (i) less sensitivity of the $\lambda 4554$ line to its variation; and (ii) less accuracy of total Ba abundance. An increase of the fraction of the odd isotopes of $\mathrm{Ba}$ from $18 \%$ to $46 \%$ leads to a decrease of $\mathrm{Ba}$ abundance derived from $\lambda 4554$ by 0.07 dex at $[\mathrm{Ba} / \mathrm{H}]=-2$ and by only 0.02 dex at $[\mathrm{Ba} / \mathrm{H}]=$ -3.3 . At the same time, the weaker subordinate line $\lambda 5853$ becomes, in fact, unmeasurable in stellar spectra.

Can we determine a desirable fraction based on only the HFS broadening of the $\lambda 4554$ line without knowing total Ba abundance? Let's imagine that we have a perfect observed profile of $\lambda 4554$. Then we fit it varying the fractional abundance of the odd isotopes of $\mathrm{Ba}$. We have modelled such situation for two "stars" using two model atmospheres.
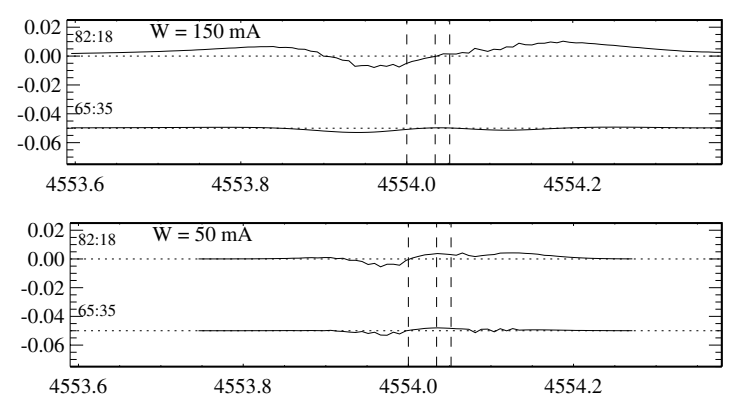

Fig. A.1. The differences between "observed" and calculated spectra, ("O"-C) in "star" 1 (top panel) and "star" 2 (bottom panel) for the evento-odd isotope ratios $82: 18$ and 65:35. In the latter case, the differences shifted down by 0.05 are shown. The HFS components of the odd isotopes appear in two groups shifted relative to the resonance line of the even isotopes at $4554.034 \AA$ by $18 \mathrm{~m} \AA$ and $-34 \mathrm{~m} \AA$. Their positions are indicated by vertical lines. See text for more details.

"Star" 1: $T_{\text {eff }}=5710 \mathrm{~K}, \log g=4.00,[\mathrm{M} / \mathrm{H}]=-0.64, V_{\text {mic }}=$ $1.1 \mathrm{~km} \mathrm{~s}^{-1},[\mathrm{Ba} / \mathrm{H}]=-0.69$. The theoretical line profile of $\lambda 4554$ calculated assuming the $\mathrm{Ba}$ odd isotope fraction of $42 \%$ and convolved with the Gaussian of $4 \mathrm{~km} \mathrm{~s}^{-1}$ and radial-tangential profile of $V_{\text {mac }}=4.5 \mathrm{~km} \mathrm{~s}^{-1}$ will serve as the "observed" line profile. Its $W_{\lambda}=152 \mathrm{~m} \AA$.

"Star" 2: $T_{\text {eff }}=6350 \mathrm{~K}, \log g=4.03,[\mathrm{M} / \mathrm{H}]=-2.07, V_{\text {mic }}=$ $1.7 \mathrm{~km} \mathrm{~s}^{-1},[\mathrm{Ba} / \mathrm{H}]=-2.14$. The "observed" profile of $\lambda 4554$ was obtained assuming the Ba odd isotope fraction of $46 \%$ and applying the Gaussian of $2 \mathrm{~km} \mathrm{~s}^{-1}$ and $V_{\mathrm{mac}}=4.7 \mathrm{~km} \mathrm{~s}^{-1}$. Its $W_{\lambda}=50 \mathrm{~m} \AA$.

Assuming the fraction of the odd isotope of $\mathrm{Ba}$, odd $=18 \%$ and then $35 \%$, we obtained the best fits for each case and for both "stars". Ba abundance and $V_{\text {mac }}$ value were allowed to vary. The Gaussian was fixed. In practice, the $V_{\text {mac }}$ values turn out to be within the uncertainty of determination: $V_{\mathrm{mac}}=4.25 \mathrm{~km} \mathrm{~s}^{-1}$ when $o d d=18 \%$ and $4.4 \mathrm{~km} \mathrm{~s}^{-1}$ when odd $=35 \%$ in "star" 1 ; $V_{\mathrm{mac}}=5.1 \mathrm{~km} \mathrm{~s}^{-1}$ when odd $=18 \%$ and $5.0 \mathrm{~km} \mathrm{~s}^{-1}$ when odd $=35 \%$ in "star" 2. The ("O"-C) differences are shown in Fig. A.1.

It can be seen from Fig. A.1 that an accuracy of line profile measurements has to be much higher than $0.05 \%$ if one wishes to determine a desirable fraction within the uncertainty of $10 \%$.

\section{References}

Anders, E., \& Grevesse, N. 1989, Geoch. Cosmochim Acta, 53, 197 Arlandini, C., Käppeler, F., Wisshak, K., et al. 1999, ApJ, 525, 886 Asplund, M., Grevesse, N., \& Sauval, A. J. 2005, ASP Conf. Ser., 336, 25 Barklem, P. S. 2006, private communication

Barklem, P. S., \& Aspelund-Johansson, J. 2005, A\&A ,435, 373

Barklem, P. S., \& O’Mara, B. J. 1998, MNRAS, 300, 863

Becker, W., \& Werth, G. 1983, Z. Phys. A, 311, 41

Bensby, T., Feltzing, S., Lundström, I., \& Ilyin, I. 2005, A\&A, 433, 185

Biehl, D. 1976, Sonderdruck der Sternwarte Kiel, 229

Blatt, R., \& Werth, G. 1982, Phys. Rev., A25, 1476

Brix, F., \& Kopfermann, H. 1952, Landolt-Börnstein, I/5 (Berlin: Springer) Burris, D. L., Pilachowski, C. A., Armandroff, T. E., et al. 2000, ApJ, 544, 302 Butler, K., \& Giddings, J. 1985, Newsletter on the analysis of astronomical spectra No. 9, University of London

Cowley, C. R., \& Corliss, C. H. 1983, MNRAS, 203, 651

Cowley, C. R., \& Frey, M. 1989, ApJ, 346, 1030

Davidson, M. D., Snoek, L. C., Volten, H., \& Dönszelmann A. 1992, A\&A, 255, 457

Fuhrmann, K. 1998, A\&A, 338, 161

Fuhrmann, K. 2004, Astron. Nachr., 325, 3

Fuhrmann, K., Pfeiffer, M., Frank, C., Reetz, J., \& Gehren, T. 1997, A\&A, 323, 909

Fulbright, J. P. 2000, AJ, 120, 1841 
Gratton, R. G., Carretta, E., Matteucci, F., \& Sneden, C. 1996, ASP Conf. Ser., 92, 307

Gratton, R. G., Carretta, E., Matteucci, F., \& Sneden, C. 2000, A\&A, 358, 671

Grevesse, N., Noels, A., \& Sauval, A. J. 1996, ASP Conf. Ser., 99, 117

Kupka, F., Piskunov, N., Ryabchikova, T. A., Stempels, H. C., \& Weiss, W. W. 1999, A\&AS, 138, 119

Kurucz, R. L., \& Peytremann, E. 1975, A Table of Semiempirical gf-values. Smithsonian Astrophys. Obs. Spec. Rep. 362

Kurucz, R. L., \& Bell, B. 1995, Atomic Line Data. Kurucz CD-ROM No. 23. Cambridge, Mass

Kurucz, R. L., Furenlid, I., Brault, J., \& Testerman. L. 1984, Solar Flux Atlas from 296 to $1300 \mathrm{~nm}$. Nat. Solar Obs., Sunspot, New Mexico

Lambert, D. L., \& Allende Prieto, C. 2002, MNRAS, 335, 325

Magain, P. 1995, A\&A, 297, 686

Magain, P., \& Zhao, G. 1993, in Origin and Evolution of the Elements. Proceedings of the Symposium, ed. N. Prantzos, E. Vangioni-Flam \& M. Casse (Cambridge University Press), 480

Magain, P., \& Zhao, G. 1993 A\&A, 268, L27

Mashonkina, L. I., \& Bikmaev, I. F. 1996, Astron. Rep., 40, 109

Mashonkina, L. I., \& Gehren, T. 2000, A\&A, 364, 249

Mashonkina, L. I., \& Gehren, T. 2001, A\&A, 376, 232
Mashonkina, L. I., Gehren, T., \& Bikmaev, I. F. 1999, A\&A, 343, 519

Mashonkina, L. I., Gehren, T., Travaglio, C., \& Borkova, T. 2003, A\&A, 397,275

Mashonkina, L. I., Kamaeva, L. A., Samotoev, V. A., \& Sakhibullin, N. A. 2004, Astron. Rep., 48, 185

McWilliam, A. 1998, AJ, 115, 1640

Mishenina, T., \& Kovtyukh, V. V. 2001, A\&A, 370, 951

Prochaska, J. X., Naumov, S. O., Carney, B. W., McWilliam, A., \& Wolfe, A. M. 2000, AJ, 120, 2513

Reader, J., Corliss, C. H., Wiese, W. L., \& Martin, G. A. 1980, Wavelengths and Transition Probabilities for Atoms and Atomic Ions, Part II, NSRDS - NBS 68, Washington, D.C.

Rutten, R. J. 1978, Sol. Phys., 56, 237

Simmerer, J., Sneden, C., Cowan, J. J., et al. 2004, ApJ, 617, 1091

Spite, M., \& Spite, F. 1978, A\&A, 67, 23

Thielemann, F.-K., Argast, D., Brachwitz, F., et al. 2002, Ap\&SS, 281, 25

Travaglio, C., Galli, D., Gallino, R., et al. 1999, ApJ, 521, 691

Truran, J. W. 1981, A\&A, 97, 391

Truran, J. W., Cowan, J. J., Pilachowski, C. A., \& Sneden, C. 2002, PASP, 114, 1293

Wujec, T., \& Weniger, S. 1981, JQSRT, 25, 167 